\title{
LA MARQUE DE LA VILLE SUR LA CAMPAGNE
}

Les campagnes ont longtemps attiré par prédilection l'attention de la géographie humaine, sans doute parce que les paysages ruraux, beaucoup plus que les paysages urbains, se prêtent à l'examen des interrelations avec le milieu physique. Aussi les célèbres grandes thèses françaises d'avant 1939 sont-elles, soit des études strictement rurales (ainsi celle de Jules Sion), soit des études régionales, mais dans lesquelles les villes tiennent peu de place, et une place à part, reléguées qu'elles sont, le plus souvent, à la fin de l'ouvrage, sans lien véritable avec l'examen approfondi des campagnes qui précède. Aussi bien ces campagnes sont-elles étudiées dans leurs aspects traditionnels, pré-industriels.

En fait deux attitudes différentes sont possibles pour le géographe placé devant un milieu rural. La première - et c'est celle qui prévalait avant la guerre - est rétrospective et génétique. On décrit des paysages et l'on explique leur formation. Il importe done d'en abstraire les adultérations récentes pour reconstituer un paysage «traditionnel», tel qu'a pu l'élaborer une certaine civilisation agraire. Or, de moins en moins, dans nos pays industrialisés, les campagnes ont conservé ce visage hérité du passé. Même lorsque leur physionomie s'est à peu près maintenue, leur économie, leur fonction, leur signification régionale se sont modifiées. Le plus souvent, la physionomie elle-même a plus ou moins profondément changé, mais un ALBERT Demangeon, lorsqu'il établissait et classait ses types d'habitats et de terroirs, éliminait systématiquement les fermes de construction moderne, les 
habitations non agricoles, les installations industrielles ou commerciales qui ont pu se plaquer sur le paysage ancien. Lorsqu'il a fait, avec la maîtrise que l'on sait, le «Tableau de la géographie de la. France», VIDAL DE LA BLACHE a recherché avant tout les aspects d'ancienneté, de continuité, de permanence.

L'autre attitude possible consiste à ne pas considérer ces apports récents comme un «abâtardissement» -.- le terme est utilisé par VIDAL dans son avant-propos-, mais comme l'indice d'une transformation en cours, d'une vocation nouvelle des campagnes, de leur association à une vie générale que la technique moderne facilite et qu'exige le développement social des ruraux. Cette attitude est résolument prospective. Elle n'exclut nullement la première, qui garde tout son intérêt en donnant la clé de ces antiques et délicates constructions humaines que sont les paysages agraires; mais, s'appuyant sur les résultats de cette étude, elle cherche à comprendre le devenir des campagnes au sein d'une civilisation de plus en plus dominée par l'industrialisation et par les villes.

Comme l'a montré Wolfgang Hartke, un même faisceau de champs laniérés cultivés en blé peut être analysé, géographiquement, de deux façons très différentes. Selon le premier éclairage, on se demandera s'il s'intégrait dans un système de champs ouverts et assolés, soumis à un régime agraire communautaire et associé à un certain type d'habitat groupé; on en reconstituera la genèse, en s'appuyant sur la forme et la disposition des champs, sur les noms de lieux-dits, sur d'éventuels textes d'archives. L'autre optique consistera à se demander qui, aujourd'hui, possède et qui exploite ces champs, et il se peut que la signification actuelle de chacun d'eux varie considérablement, selon qu'il dépend d'une exploitation agricole de type polyculteur ancien, ou d'une ferme spécialisée en blé, ou encore d'un ouvrier d'industrie qui ne trouve là qu'un appoint et qui, le jour où son salaire s'améliorera, le laissera simplement en friche, en attendant, peut-être, d'y construire sa maison.

Ces transformations récentes sont presque toujours liées aux processus de l'urbanisation. Le développement économique et social s'accompagne d'une intégration de plus en plus poussée des différents milieux, ruraux et urbains, qui constituent ce que nous appelons une «région». Héritées d'antiques civilisations agraires, les campagnes des pays industrialisés apparaissent aujourd'hui comme des composantes, parmi d'autres, d'un complexe régional, et dans cet ensemble hétérogène, mais cohérent, qu'est la région, l'élément moteur est évidemment la ville: elle fournit généralement l'essentiel des capitaux, des machines, des emplois non agricoles, des lieux de recherche et de formation professionnelle; elle est, avant tout, le centre coordinateur de l'ensemble. Rechercher la marque de la ville sur la campagne constitue donc un des aspects essentiels de cette façon nouvelle d'appréhender les faits ruraux.

Ce n'est guère qu'après la dernière guerre que la géographie française s'est préoccupée d'analyser de près ces rapports. Déjà, certes, en 1922 l'historien Gaston RoupneL avait publié un ouvrage sur «La ville et la campagne au XVII ${ }^{P}$ siècle», consacré à la région de Dijon et ce livre est un modèle de géographie rétrospective. Mais la transposition de cette méthode à l'époque actuelle n'apparaît qu'avec la deuxième Semaine sociologique, organisée par GEORGES FRIEDMANN en 1951, sur le thème «villes et campagnes». Depuis lors, les travaux se sont multipliés qui montrent l'action de la ville sur la campagne, à la fois comme moteur et comme frein. Citons notamment la thèse de JEAN LABAsse intitulée «Les capitaux et la région» (1955), celle de BERNARD KAYSER sur «Campagnes et villes de la Côte d'Azur» (1960), celle de RAYMOND DUGRAND sur «Villes et campagnes en Bas-Languedoc» (1963).

Mais sous l'influence de la ville les campagnes peuvent, soit progresser, soit se dégrader. Les marques négatives de l'action urbaine sont même celles qui frappent le plus. C'est d'abord la diminution et le vieillissement de la population rurale, entraînant une sclérose des structures agraires; c'est aussi, même sans dépeuplement, la friche d'origine sociale, née de la transformation sociomprofessionnelle de la population rurale; c'est la spéculation foncière péri-urbaine stérilisant l'espace agricole dans une auréole qui entoure d'autant plus largement la ville que celle-ci grandit plus vite; c'est enfin le grignotage et la submersion de l'espace agricole par les aménagements urbains et industriels. 
Cependant les marques positives ne manquent pas: l'agriculture est stimulée par l'essor de marchés de consommation de plus en plus larges et mieux reliés à la production; les banlieues maraîchères, première forme, très ancienne, de ces rapports, font place à une organisation multirégionale de la production agricole qui permet à chaque contrée d'adopter la spécialisation qui convient le mieux à ses aptitudes. L'industrie se répand dans les campagnes, d'abord sous la forme d'industrie proprement rurale, à domicile, puis par le desserrement des usines qui se trouvent trop à l'étroit dans les banlieues urbaines. La ville elle-même explose et essaime des complexes résidentiels dans des campagnes dont on préfère le calme et le bon air, à condition d'être commodément relié par autoroute à la ville. Plus loin encore s'aménagent des zones de détente, des «banlieues du dimanche», des résidences secondaires. Certaines zones forestières cessent d'être intégrées à l'économie rurale pour devenir des parcs ou des réserves naturelles; certains villages peuvent même se muer en véritables «musées de plein air». Par-dessus tout, les ruraux sortent de leur isolement et participent de plus en plus aux avantages dont jouissaient déjà les citadins: loisirs culturels et sportifs, possibilités de faire des études variées, mobilité professionnelle, chances d'ascension sociale. C'est cela, et non une submersion de l'espace rural par une banlieue gigantesque, qu'il faut entendre par l'expression «urbanisation des campagnes».

Mais ces différentes formes, positives ou négatives, de l'action des villes sur les campagnes comportent des dosages et des rythmes variés, selon la nature des liens existants entre les deux milieux géographiques. L'action des villes sur les campagnes peut revêtir trois formes principales, qui dépendent essentiellement du type de ville dont il s'agit et de l'attitude des citadins à l'égard des campagnes.

\section{La Stimulation Agricole}

Que la ville soit entourée d'une auréole d'agriculture plus intensive, c'était vrai déjà de l'ère pré-industrielle et un économiste comme voN THÜNEN en avait fait la théorie voici plus de cent ans. Mais il importe d'examiner de près les mécanismes de cette stimulation. Celle-ci peut être de nature purement commerciale, sans intervention directe des citadins dans la production agricole. Ainsi les villes de l'espace rhénan, qui constituent depuis le Moyen Age un des semis urbains les plus denses du monde, ont amené les campagnes voisines à se spécialiser précocement — dès le $x_{1 I I}{ }^{\mathrm{e}}$ siècle — dans la production des céréales panifiables, destinées à la consommation urbaine. Mais c'était là une stimulation purement extérieure, car les citadins se désintéressaient de la propriété foncière rurale, ayant dans le grand commerce et dans l'industrie des sources de revenus autrement fructueuses. De là la prospérité ancienne de cette société de paysans propriétaires; de là aussi ses difficultés actuelles, dues au trop grand émiettement de la structure agraire et à l'insuffisance des investissements qu'exigerait la modernisation.

$\mathrm{Au}$ contraire, dans le Bas-Languedoc décrit par R. DuGRAND, les citadins du XIX ${ }^{e}$ siècle se sont détournés de l'industrie et du grand commerce pour faire de la propriété foncière rurale leur spéculation majeure. Grâce à leurs investissements, cette plaine jusque là vouée à la polyculture méditerranéenne a pu devenir le plus grand vignoble du monde. Mais il est anormai de voir des villes comme Montpellier ou Béziers vivre essentiellement de la rente du sol. Cette symbiose touche au parasitisme. La ville avait été un moteur lorsqu'elle avait réalisé, voici cent ans, une conversion que jamais le petit paysan n'aurait pu faire seul; aujourd'hui, la part qu'elle prélève du bénéfice agricole apparaît de moins en moins justifiée.

\section{LA StÉRILISATION DEs CAMPagnes}

Dans d'autres cas l'évolution récente a accentué, au contraire, le divorce entre la ville et son environnement rural. Deux cas peuvent être distingués. D'abord la stérilisation directe, par l'effet de la spéculation foncière péri-urbaine, non pas en vue de l'intensification agricole, mais pour profiter de la plus-value des terrains à bâtir. B. KAYSER a décrit autour des villes de la Côte d'Azur des halos de campagnes véritablement «stérilisées» par les immobilisations foncières des citadins. Ceux-ci, dont beaucoup appartiennent à une grande 
bourgeoisie internationale qui vit là un peu comme dans une colonie, ne s'intéressent nullement à la production agricole et la polyculture traditionnelle meurt pour n'être remplacée que par la friche ou par les parcs de somptueuses résidences. A plus grande échelle, les grandes villes américaines s'entourent aussi d'une ceinture de friches qui atteint souvent plusieurs kilomètres de profondeur.

Dans d'autres cas la stérilisation est indirecte, sans spéculation foncière de la part des citadins. C'est le cas du développement récent et rapide de tels complexes industriels comme la sidérurgie lorraine ou comme l'ensemble de Lacq, dans le Béarn: des usines ultra-modernes, distribuant de hauts salaires et offrant des logements confortables dans des cités toutes neuves sont brutalement plaquées sur des campagnes archaïques. Celles-ci, loin d'en être stimulées, voient généralement s'accélérer leur processus déjà amorcé de décadence; la friche s'étend, en attendant que naisse, peutêtre, une agriculture toute nouvelle, mais qui sera généralement introduite par de nouveaux venus.

Dans ces divers cas il s'agit, on le voit, de villes qui se désintéresscnt de leur environnement rural, qui leur tournent le dos, qui se développent comme des «îles» dans un milieu qu'elles n'associent pas à leur croissance.

L'ASSOCIATION Ȧ UN DÉVELOPPEMENT GLOBAL

Le processus le plus sain est, évidemment, celui qui entraîne villes et campagnes dans un même développement global. Il semble qu'il faille pour cela, au départ, des campagnes assez densément peuplées et solidement structurées, mais sans emprise foncière citadine; un développement industriel précoce et continu, qui crée un appel de main-d'œuvre rurale et d'amples migrations pendulaires; un essaimage précoce des usines dans les campagnes; une adaptation de l'agriculture aux marchés de vente; bref, une association des villes et des campagnes sans sujétion de celles-ci à celles-là, des campagnes «urbanisées» sortant, sans hiatus et sans crise, de l'ancienne dualité villes-campagnes. Des pays comme le Danemark, la Hollande, la Suisse, des régions comme celles du Rhin moyen ou du Bas-Rhône ont jusqu'ici le mieux réussi ce passage d'une civilisation agraire traditionnelle à un développement économique et social généralisé. Réaliser cette mutation, mettre fin à l'isolement des ruraux est une des grandes entreprises des temps modernes. Dans la mesure où elle met en jeu des facteurs spatiaux, le géographe peut aider les responsables d'une politique de développement à préciser les diagnostics et à choisir les mesures qui s'imposent.

ETIENNE JUILLARD 